\title{
Evaluación de la influencia de la agitación para la producción de biomasa microalgal en un fotobiorreactor panel plano a escala laboratorio
}

\section{Evaluation of the Influence of Agitation for the Production of Microalgal Biomass in a Flat Panel Photobioreactor at Laboratory Scale}

\author{
Jennifer L. Doncel Núñez \\ Ingeniera química, Fundación Universidad de América, Colombia \\ https://orcid.org/0000-0003-4618-6317 | jennifer.doncel0205@hotmail.com \\ Heidy L. Moreno Saboyá \\ Ingeniera química, Fundación Universidad de América, Colombia \\ https://orcid.org/0000-0002-6937-5084 | heidylilianamoreno@gmail.com
}

\section{RESUMEN}

Se evaluó la influencia de la agitación sobre la producción de biomasa en un fotobiorreactor (FBR) panel plano mediante la modificación de los aspersores en el difusor. La evaluación se realizó mediante la elaboración de 3 difusores con diámetros de aspersor diferentes $(1,2$ y $4 \mathrm{~mm})$. A través de un análisis de varianza ANOVA y una prueba $t$, se seleccionaron los diámetros de 1 y $2 \mathrm{~mm}$ para ingresar dióxido de carbono $\left(\mathrm{CO}_{2}\right)$, obteniendo una tasa de crecimiento de 0,37 días $^{-1}$ y 0,35 días $^{-1}$, respectivamente. El análisis indicó que a menor diámetro de aspersor en el difusor mayor tasa de crecimiento. Mediante el planteamiento de las ecuaciones de transferencia de masa se verificó que la disminución del tamaño de la burbuja promueve la transferencia entre las fases gas-líquido.

Palabras clave: microalgas, fotobiorreactores panel plano, agitación, difusor, dióxido de carbono. 


\begin{abstract}
The influence of agitation on the production of biomass in a flat panel photobioreactor was evaluated by modifying the sprinklers in the diffuser. The evaluation was carried out through the elaboration of 3 diffusers with different sprinkler diameters $(1,2$, and $4 \mathrm{~mm})$. The sprinklers with diameters of 1 and $2 \mathrm{~mm}$ were selected for the entry of carbon dioxide $\left(\mathrm{CO}_{2}\right)$ via ANOVA and $t$-test, obtaining a growth rate of 0.37 days ${ }^{-1}$ and 0.35 days ${ }^{-1}$, respectively. The analysis showed that the smaller diameter of the sprinkler in the diffuser, the higher the growth rate. Besides, by considering the mass transfer equations it was verified that the decrease in the size of the bubble promotes the transfer within the gas-liquid phases.
\end{abstract}

Keywords: Microalgae, flat panel photobioreactors, agitation, diffuser, carbon dioxide.

\section{INTRODUCCIÓN}

Para mejorar las condiciones de crecimiento de la microalga Chlorella vulgaris se han desarrollado diferentes proyectos, tales como la evaluación del crecimiento desde varios tipos de fotobiorreactores (tipos placa plana y airlift). Sin embargo, no se ha llevado a cabo un análisis con respecto a la agitación. Por ello, esta investigación busca proponer e incluir este factor en el cultivo de Chlorella vulgaris, al ser una alternativa prometedora en procesos de biorremediación de $\mathrm{CO}_{2}$ que garantiza la mejor distribución de la microalga en el medio cultivo.

Teniendo en cuenta que las microalgas en fotobiorreactores de placa plana poseen altas eficiencias fotosintéticas, pero al mismo tiempo presentan problemas en el control de difusión de $\mathrm{CO}_{2}$ relacionados principalmente con la transferencia de masa (Soo \& Chould-Gyun, 2003), la evaluación de la variable agitación resulta necesaria. El hecho de que la agitación se relacione con la transferencia afecta la disponibilidad de $\mathrm{CO}_{2}$ para la microalga Chlorella vulgaris y, por lo tanto, la producción de biomasa en este tipo de fotobiorreactores.

Para la evaluación del parámetro agitación en este tipo de fotobiorreactores se hizo necesario evaluar el diseño de los difusores (tubo perforado) que involucran aspectos como cantidad de burbujas y diámetro de burbuja (Ladislav et al., 2010) desde la agitación neumática utilizada. Los aspectos antes mencionados fueron objeto de estudio en la presente investigación, la cual se enfoca en el parámetro agitación debido a su efecto en la transferencia de masa, variable que afecta la disponibilidad de nutrientes e influye en un nivel de producción más elevado al proporcionar un suministro adecuado de $\mathrm{CO}_{2}$ a través de la interface cultivo-burbuja y la eliminación de $\mathrm{O}_{2}$ por generación de turbulencia en el medio (St-Onge, 2013). Aunque el parámetro agitación no ha sido considerado en otras evaluaciones, este es una variable condicionante en la producción de biomasa microalgal.

\section{METODOLOGÍA}

La cepa microalgal empleada en esta investigación es Chlorella vulgaris, la cual fue suministrada por el grupo de investigación BIOTECFUA de la Fundación Universidad de América y se encontraba en medio líquido en tubos de ensayo, siendo un back up de la cepa pura previamente adquirida por otros grupos. Se utilizó como medio de cultivo, agua y el fertilizante agrícola foliar Foliagro en una proporción de 2:1000. El medio de cultivo fue usado desde la fase de incubación hasta el escalamiento al fotobiorreactor, el cual fue esterilizado a una temperatura de $120^{\circ} \mathrm{C}$ durante 40 minutos previo a su uso.

La microalga se inoculó y se incubó implementando una incubadora que cumplía con las siguientes condiciones: temperatura óptima de $25^{\circ} \mathrm{C}$, fuente lumínica de halógeno de $8 \mathrm{~W}$ de color rojo y un fotoperiodo $12 / 1$.

El escalamiento a partir del inóculo inició con un volumen de $10 \mathrm{~mL}$ de microalga y $30 \mathrm{~mL}$ de medio de cultivo, al cual se le agregó $50 \mathrm{~mL}$ de medio de cultivo hasta alcanzar un volumen de $500 \mathrm{~mL}$. Posteriormente, se agregó $100 \mathrm{~mL}$ de medio de cultivo hasta 
alcanzar $1 \mathrm{~L}$ y por último, $150 \mathrm{~mL}$ hasta alcanzar $3 \mathrm{~L}$, tras lo cual se procedió con su escalamiento al fotobiorreactor de panel plano.

El desarrollo de esta investigación se enfocó en dos etapas: una fase preexperimental en la cual se seleccionaron los diámetros de aspersor en los tubos difusores con respecto a las curvas de crecimiento, seguida de una fase experimental en la que se evaluó el crecimiento al adicionar una mezcla de $\mathrm{CO}_{2}$ y aire como fuente de agitación tras emplear los diámetros seleccionados.

\section{Fase preexperimental}

En esta fase se llevó a cabo la construcción de dos fotobiorreactores de panel plano en polimetil-metacrilato (PMMA), los cuales contaban con un ducto desgasificador y un termostato en su interior. La figura 1 muestra uno de los fotobiorreactores empleados.

El dimensionamiento de los difusores implicó el conocimiento tanto del diámetro de los aspersores como el espaciamiento entre estos. Lo anterior se calculó mediante la ecuación 1 , en la que se consideró la misma longitud $(0,392 \mathrm{~m})$ y cantidad de aspersores.

Distancia entre aspersores

$=\frac{L-(\text { Cantidad de aspersores } * \text { Diámetro del aspersor })}{\text { Cantidad de aspersores }+1}$

(Ec. 1)

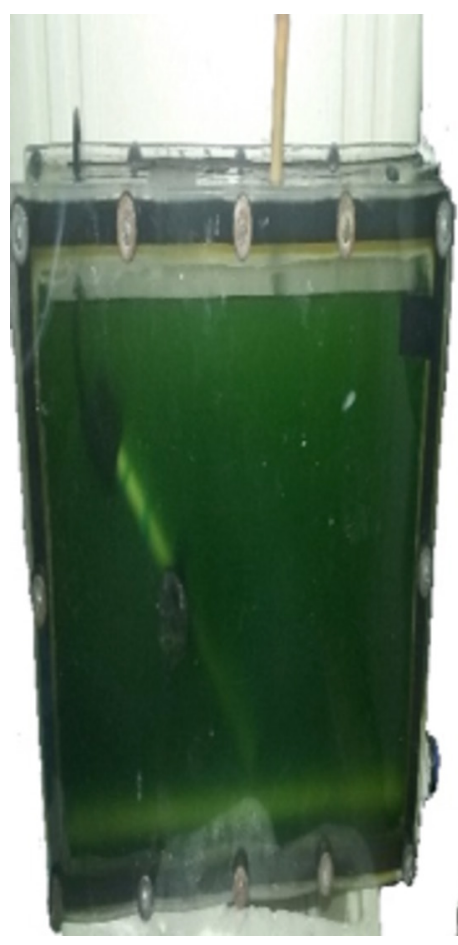

Figura 1. Fotobiorreactor panel plano Fuente: elaboración propia.

Los tubos difusores fueron elaborados en policloruro de vinilo (PVC) y se adicionaron a las láminas laterales del fotobiorreactor empleando silicona para vidrios. Estos tubos se cambiaban de forma manual conforme al diámetro con el cual se iba a experimentar. La figura 2 (izquierda) muestra las dimensiones del equipo y las características del tubo difusor (derecha).

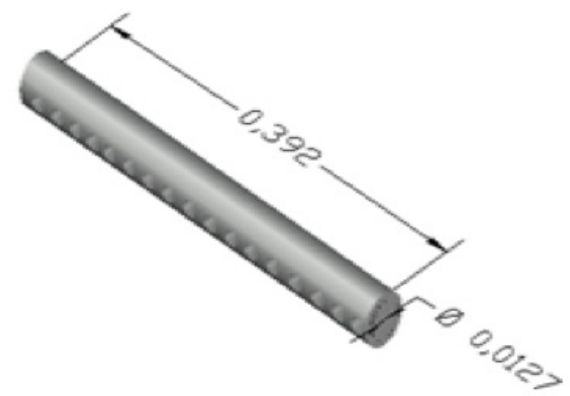

Figura 2. Dimensiones del fotobiorreactor panel plano y los tubos difusores en metros (m) Fuente: elaboración propia.

Por otra parte, la ecuación que permitió determinar si el equipo resultaba adecuado para el proceso fotosintético fue la relación superficie-volumen (ecuación 2) cuyas unidades son $\mathrm{m}^{-1}$ (Rubio-Fernández \& Hernández, 2016): 
relación sup - volu $=\frac{\text { área de la placa frontal }}{\text { volumen de equipo }}=\frac{0.08}{0.004}=20 \mathrm{~m}^{-1}$ (Ec. 2$)$

El valor de la relación superficie-volumen cumplió con el rango entre 20 y $200 \mathrm{~m}^{-1}$ para la adecuación de luz, por lo que el equipo presentaba buen acceso a la luz, permitiendo que el inóculo realizara el proceso fotosintético sin dificultades.

El montaje del sistema de la etapa preexperimental contaba con una bomba de aire marca Resun AC9904 de 4 salidas que se conectaba a un filtro de trompo mediante mangueras de $6 \mathrm{~mm}$ de espesor; este filtro permitió garantizar la esterilidad del aire que entraba al equipo. Luego, se conectó un manómetro Ritherm ${ }^{\circ}$ $2 \frac{1}{2}$ " que permitió conocer la presión del sistema, la cual se regulaba mediante un sistema de llaves ubicadas a la salida del manómetro. Las mangueras que salían de estas llaves fueron conectadas a los extremos laterales del fotobiorreactor.

Se realizó el conteo celular de microorganismos en un microscopio marca BioBlue mediante una cámara Neubauer durante 15 días (para un mayor período de estudio) cada 24 horas para cada diámetro, obteniendo 3 réplicas de cada diámetro. El número de células por $\mathrm{mL}$ se determinó mediante la ecuación 3 , donde el volumen de la cámara es de $0,1 \mathrm{~mm}^{3}$ y los cuadrantes totales son 25 (Hernández, 2014).

$$
\frac{\text { Células }}{m L}=\frac{\text { número de células } * \text { factor de dilución }}{\text { volumen } * \frac{\text { cuadrantes contados }}{\text { cuadrantes totales }}} * \frac{1000 \mathrm{~mm}^{3}}{1 \mathrm{~mL}} \text { (Ec. 3) }
$$

\section{Análisis estadístico}

Las curvas de crecimiento de cada difusor fueron evaluadas bajo un análisis de varianza (ANOVA) de un factor con el fin de conocer si existían diferencias significativas en las tasas de crecimiento celular como resultado de los diámetros del aspersor. Las hipótesis planteadas fueron las siguientes:

Hipótesis nula (Ho): no existe una diferencia significativa en las tasas de crecimiento por el cambio en el diámetro en los aspersores.

Hipótesis alterna (Ha): existe una diferencia significativa en las tasas de crecimiento por el cambio en el diámetro en los aspersores.
La hipótesis nula fue aceptada utilizando como criterio de comparación el valor registrado de $F$ con respecto al valor de $F$ crítico a un nivel de significancia de 0,05 . Por lo anterior, y como segundo tratamiento estadístico, se procedió a desarrollar la comparación entre parejas para simplificar los niveles (diámetros) y encontrar aquellos que afectaban la variable respuesta (tasas de crecimiento) mediante la comparación del valor de la probabilidad de dos colas $P(T<=t)$ y la probabilidad de la tabla ANOVA.

\section{Fase experimental}

En esta fase se emplearon los diámetros de aspersor de 1 y $2 \mathrm{~mm}$ en los tubos difusores que fueron seleccionados en la etapa preexperimental mediante la prueba $t$ student.

Antes de iniciar la fase experimental se seleccionó una concentración de $\mathrm{CO}_{2}$ de $9 \% \mathrm{CO}_{2}$ y $91 \%$ aire.

El montaje del sistema de la parte experimental (figura 3) involucró el mismo montaje de la parte preexperimental, adicionando un cilindro de $6 \mathrm{~m}^{3}$ con mezcla ( $9 \% \mathrm{CO}_{2} \mathrm{v} / \mathrm{v}$ ) y una tarjeta K-33 ICB como sensor para verificar el porcentaje de $\mathrm{CO}_{2}$ que entraba al fotobiorreactor de panel plano.

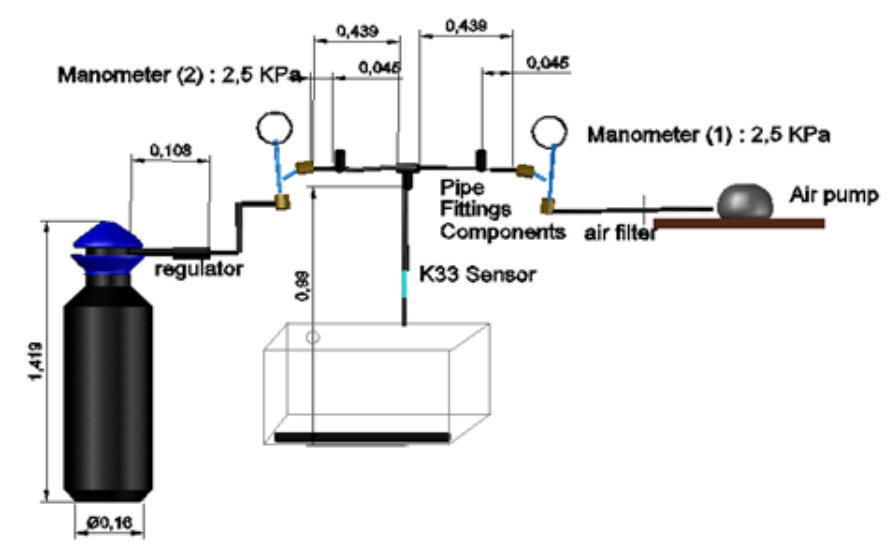

Figura 3. Montaje del sistema experimental y dimensiones de las conexiones $(\mathrm{m})$

Fuente: elaboración propia.

Haciendo uso del sistema antes descrito, se llevó a cabo la agitación por períodos de 6 y 18 horas con mezcla y aire, respectivamente, manteniendo el caudal fijo mediante un regulador de flujo que manejaba un rango de 0 a $4 \mathrm{~L} / \mathrm{min}$. 
Teniendo en cuenta que las microalgas muestran una gran dependencia respecto al $\mathrm{pH}$, el cual varía conforme a la cantidad de $\mathrm{CO}_{2}$ disuelto, este fue monitoreado mediante un potenciómetro marca Milwaukee (figura 4), que registró un valor máximo de 7,5. Para esta fase, el conteo celular se llevó a cabo en una cámara Neubauer durante 10 días para los diámetros de 1 y $2 \mathrm{~mm}$, llevando a cabo 3 réplicas de cada uno. EI conteo se realizó durante el tiempo de agitación con el cilindro de $\mathrm{CO}_{2}$. El número de células por $\mathrm{mL}$ se determinó nuevamente con la ecuación 2 , incorporando esta vez el factor de dilución debido a que se realizaron diluciones seriadas con un factor de 1:10.

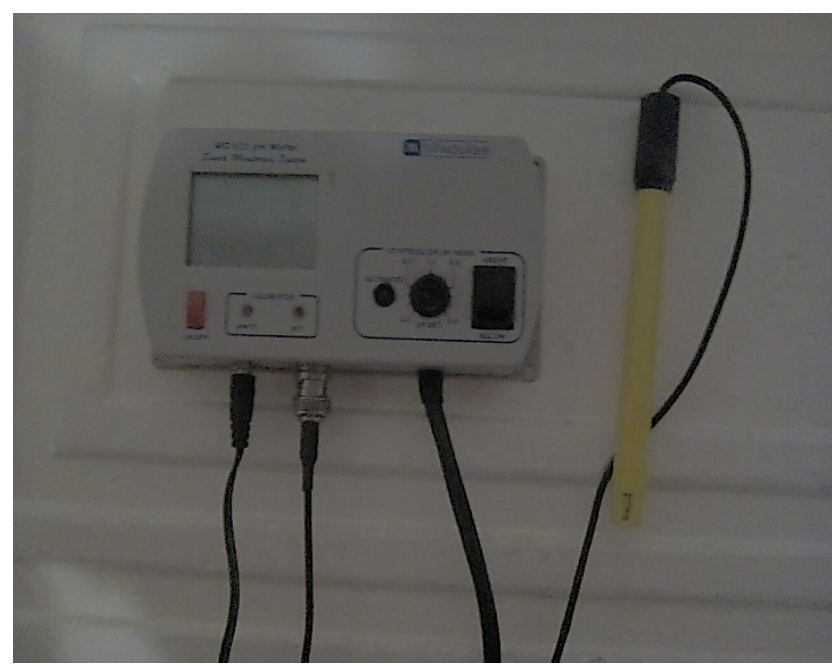

Figura 4. Potenciómetro marca Milwaukee Fuente: elaboración propia.

Haciendo uso de parámetros poblaciones como la velocidad específica de crecimiento, también conocida como tasa de crecimiento $(\mu)$ (Arredondo et al., 2007), y en vista de que las microalgas se reproducen mediante división binaria, la ecuación 4 fue utilizada para el análisis de resultados.

$$
\mu=\frac{\left[\frac{\ln \left(\frac{x_{2}}{x_{1}}\right)}{\ln (2)}\right]}{\left(t_{2}-t_{1}\right)}
$$

\section{RESULTADOS Y ANÁLISIS}

Se dimensionaron los tres difusores con los respectivos diámetros de aspersor (1,2 y $4 \mathrm{~mm}$ ). Para este fin, se calculó la distancia entre un aspersor y otro, la cual depende primordialmente de la longitud del tubo y de la cantidad de aspersores totales con los que cuenta el difusor. Por lo anterior, se aplicó la ecuación 1 para cada tubo difusor. La figura 5 presenta el resultado final de cada difusor.
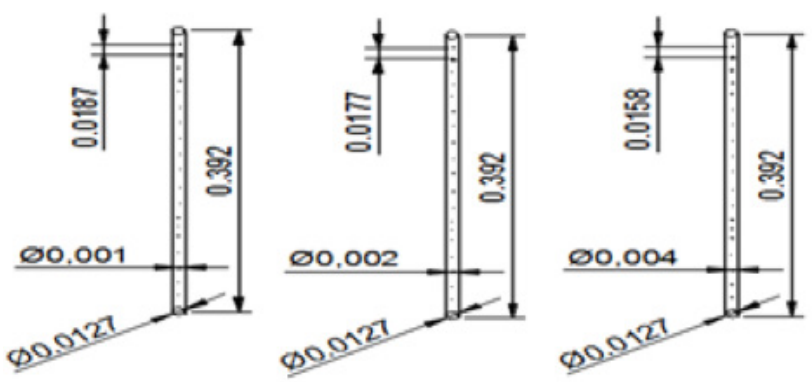

Figura 5. Dimensionamiento del espaciamiento entre los aspersores de los tubos difusores $(\mathrm{m})$

Fuente: elaboración propia.

\section{Fase preexperimental}

\section{Presión de entrada de aire al sistema}

Luego de la elaboración de los difusores, con sus respectivos diámetros, se procedió a calcular la presión de entrada de aire al difusor, la cual influye en la formación de la burbuja. Esta se calculó haciendo uso de la ecuación de continuidad y la ecuación de Bernoulli.

Los valores necesarios tanto para la ecuación de continuidad como para la ecuación de Bernoulli se reportan en la tabla 1 y 2 , respectivamente, con lo cual se obtuvo que la velocidad 2 y la presión 2 tienen un valor de $0,0665 \mathrm{~m} / \mathrm{s}$ y $2.511,60 \mathrm{~Pa}$, respectivamente.

Tabla 1. Valores necesarios para la ecuación de continuidad

\begin{tabular}{ccc}
\cline { 2 - 3 } & Manguera & Difusor \\
\cline { 2 - 3 } Velocidad del aire $(\mathrm{m} / \mathrm{s})$ & 0,4272 & \\
\hline Área transversal $\left(\mathrm{m}^{2}\right)$ & $1,96 * 10^{-5}$ & $1,26 * 10^{-4}$ \\
\hline
\end{tabular}

Fuente: elaboración propia 
Tabla 2. Valores ecuación de Bernoulli

\begin{tabular}{cc}
\hline Variable & Magnitud \\
\hline$Z_{1}$ & $0,99 \mathrm{~m}$ \\
$Z_{2}$ & $0 \mathrm{~m}$ \\
$P_{1}$ & $2500 \mathrm{~Pa}$ \\
$\rho$ & $1,185 \mathrm{Kg} / \mathrm{m}^{3}$ \\
$g$ & $9,8 \mathrm{~m} / \mathrm{s}$ \\
\hline
\end{tabular}

Fuente: elaboración propia.

\section{Curvas de crecimiento con aire}

Teniendo en cuenta el promedio de cada una de las réplicas por día obtenidas mediante la ecuación 2, a las cuales se aplicó el logaritmo natural para cada concentración, se obtuvieron los resultados señalados en la figura 6.

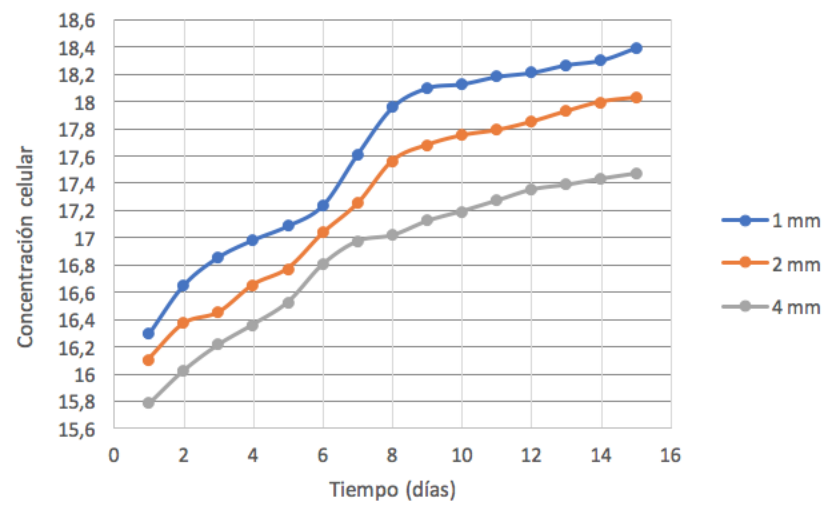

Figura 6. Curvas de crecimiento de Chlorella vulgaris con los diferentes diámetros de aspersor

Fuente: elaboración propia.

Como se aprecia en la figura 6, la curva de $4 \mathrm{~mm}$ posee una fase exponencial que se prolonga desde el día 5 hasta el día 7. En contraste, la fase exponencial de la curva de $2 \mathrm{~mm}$ se ve reflejada entre los días 6 y 9. Por último, la curva de $1 \mathrm{~mm}$ presenta una fase exponencial más prolongada que inicia en el día 6 y se extiende hasta el día 10.

A partir de la investigación desarrollada por Mohsenpour y Willoughby (2015), que muestra la tasa de crecimiento para un cultivo con aireación de 99,97 $\%$ de aire y $0,03 \%$ de $\mathrm{CO}_{2}$, se encuentra que los resultados reportados por estos autores corresponden al doble de la mejor tasa de crecimiento obtenida en la presente investigación, la cual se registró con el diámetro de $1 \mathrm{~mm}$, como se aprecia en la tabla 3. Esto se debió a la cantidad de flujo de aire que afecta el crecimiento, puesto que para obtener una alta tasa de crecimiento a volúmenes de cultivo similares se debe emplear un flujo de 4,44 vvm, como reporta el trabajo de los autores antes mencionados, quienes obtuvieron buenos resultados utilizando dicho flujo y bajo la incidencia de la luz roja. En este sentido, y por cuestiones económicas, esta investigación empleó un flujo de 0,17 vvm, que resulta menor al flujo anteriormente mencionado, lo cual, en efecto, provocó que la fluidización no fuese suficiente para que cada una de las microalgas recibiera la misma cantidad de luz proveniente de las lámparas de halógeno de luz roja. Por lo anterior, se produjo una desaceleración en el crecimiento del cultivo.

Tabla 3. Tasas de crecimiento

\begin{tabular}{ccc}
\hline $\begin{array}{c}\text { Diámetro } \\
(\mathrm{mm})\end{array}$ & $\begin{array}{c}\text { Tasa de crecimiento } \\
\text { propia } \\
\left(\text { dias }^{-1}\right)\end{array}$ & $\begin{array}{c}\text { Tasa de crecimiento } \\
\text { Mohsenpour y } \\
\text { Willoughby [2015] } \\
\left(\text { dias }^{-1}\right)\end{array}$ \\
\hline 1 & 0,217 & 0,42 \\
\hline 2 & 0,198 & - \\
\hline 4 & 0,174 & - \\
\hline
\end{tabular}

Fuente: elaboración propia.

Por otro lado, los valores promedio de las tasas de crecimiento permitieron apreciar que bajo los diámetros de aspersor de 1 y $2 \mathrm{~mm}$ se presentó una mayor velocidad de crecimiento con respecto al diámetro de $4 \mathrm{~mm}$. Lo anterior fue puesto a prueba posteriormente con el análisis de varianza ANOVA y la prueba t.

El análisis estadístico se llevó a cabo con una tabla ANOVA de un factor donde se compararon las tasas de crecimiento con respecto a los diámetros de aspersor en el difusor, utilizando un valor de significancia de 0,05. La tabla 4 presenta los resultados de los análisis de varianza indicando que no existe una diferencia significativa en las tasas de crecimiento al obtener un valor de $F$ menor al $F$ crítico $(3,2199)$. 
Tabla 4. Resultados análisis de varianza ANOVA

\begin{tabular}{ccccccc}
\hline $\begin{array}{c}\text { Origen de las } \\
\text { variaciones }\end{array}$ & $\begin{array}{c}\text { Suma de } \\
\text { cuadrados }\end{array}$ & $\begin{array}{c}\text { Grados de } \\
\text { libertad }\end{array}$ & $\begin{array}{c}\text { Promedio de los } \\
\text { cuadrados }\end{array}$ & F & Probabilidad & Valor crítico para F \\
\hline Entre grupos & 0,013694307 & 2 & 0,006847153 & 0,346894226 & 0,70888621 & 3,219942293 \\
\hline $\begin{array}{c}\text { Dentro de los } \\
\text { grupos }\end{array}$ & 0,829014783 & 42 & 0,019738447 & & \\
\hline Total & $\mathbf{0 , 8 4 2 7 0 9 0 8 9}$ & $\mathbf{4 4}$ & & & \\
\hline
\end{tabular}

Fuente: elaboración propia.

En vista de los resultados obtenidos, se realizó una comparación entre parejas para simplificar los diámetros y encontrar aquellos que afectaban las tasas de crecimiento. Mediante el método de Dunnet, se obtuvo que los diámetros de 1 y $2 \mathrm{~mm}$ presentaban cambios significativos debido a la comparación de probabilidad de 2 colas con respecto a la probabilidad de la tabla ANOVA, la cual es mayor, como se aprecia en la tabla 5. Por ende, se determinó que estos diámetros ( 1 y $2 \mathrm{~mm}$ ) eran adecuados para llevar acabo la fase experimental.

Tabla 5. Análisis de varianzas desiguales entre los grupos de $1 \mathrm{~mm}$ y $2 \mathrm{~mm}$

\begin{tabular}{|c|c|c|}
\hline & $1 \mathrm{~mm}$ & $2 \mathrm{~mm}$ \\
\hline Media & 0,217071218 & 0,197806213 \\
\hline Varianza & 0,032387217 & 0,01831539 \\
\hline Observaciones & 14 & 14 \\
\hline Diferencia hipotética de las medias & 0 & - \\
\hline Grados de libertad & 24 & - \\
\hline Estadístico $t$ & 0,320123824 & - \\
\hline $\mathrm{P}(\mathrm{T}<=\mathrm{t})$ una cola & 0,375822423 & - \\
\hline Valor crítico de t (una cola) & 1,71088208 & - \\
\hline $\mathrm{P}(\mathrm{T}<=\mathrm{t})$ dos colas & 0,751644847 & - \\
\hline Valor crítico de $t$ (dos colas) & 2,063898562 & - \\
\hline
\end{tabular}

Fuente: elaboración propia.

\section{Fase experimental}

Utilizando la ecuación de continuidad y la ecuación de Bernoulli se calculó la presión con la que la mezcla de $\mathrm{CO}_{2}$ ingresaría al difusor. Debido a que la densidad de la mezcla en la parte experimental varía con respecto a la densidad de la fase preexperimental (aire) fue necesario calcular la densidad de la mezcla con un valor de $1,25 \mathrm{Kg} / \mathrm{m}^{3}$. 
Con la densidad calculada anteriormente y los valores que se presentan en la tabla 6 se halló la presión a la entrada del difusor. Todas las variables de esta ecuación se mantuvieron constantes con respecto a la fase preexperimental, exceptuando la densidad. El resultado obtenido señala que la presión 2 debe ser de 2.534,19 Pa. Teniendo en cuenta este resultado, se observó que el valor de $\mathrm{P}_{2}$ de la fase preexperimental reportó una diferencia porcentual de 0,00892 con respecto al valor de $P_{2}$ de la fase experimental. Por lo anterior, este valor no presento una diferencia significativa.

Tabla 6. Valores ecuación de Bernoulli

\begin{tabular}{cc}
\hline Variable & Magnitud \\
\hline$Z_{1}$ & $0,99 \mathrm{~m}$ \\
\hline$Z_{2}$ & $0 \mathrm{~m}$ \\
\hline$P_{1}$ & $2500 \mathrm{~Pa}$ \\
\hline$p$ & $1,25 \mathrm{~kg} / \mathrm{m}^{3}$ \\
\hline$g$ & $9,8 \mathrm{~m} / \mathrm{s}$ \\
\hline
\end{tabular}

Fuente: elaboración propia.

\section{Curvas de crecimiento con $\mathrm{CO}_{2}$}

Utilizando los diámetros de aspersor seleccionados en la fase preexperimental ( 1 y $2 \mathrm{~mm}$ ) se procedió a desarrollar la curva de crecimiento de la microalga, la cual se representó con el promedio de cada una de las réplicas por día. A dichas replicas, a su vez, se les aplicó el logaritmo natural para cada concentración.

Por un lado, con el diámetro de $2 \mathrm{~mm}$ se obtuvo una tasa de crecimiento de 0,35 dias ${ }^{-1}$ (10 días de cultivo), la cual resulta superior a la reportada por Suárez-Chaparro y Chicagui (2014), quienes utilizaron el mismo porcentaje de mezcla e igual diámetro y obtuvieron un valor promedio de tasa de crecimiento de 0,18 días $^{-1}$ (5 días de cultivo). Lo anterior se debe a que la presente investigación consideró los caudales a suministrar al difusor en contraste con la investigación realizada por estos autores, lo que influenció la manera en que se llevó a cabo la mezcla. Por otra parte, con un diámetro de aspersor de $1 \mathrm{~mm}$ se obtuvo una tasa de crecimiento de 0,37 dias ${ }^{-1}$, que es superior a la registrada con el diámetro de $2 \mathrm{~mm}$.

El incremento celular registrado se debe a que las burbujas formadas con el diámetro de aspersor de $1 \mathrm{~mm}$ subían a baja velocidad, haciendo que estas permanecieran más tiempo en el cultivo. De esta forma, el $\mathrm{CO}_{2}$ que se encontraba en la burbuja tenía más tiempo para disolverse. La figura 7 muestra las curvas de crecimiento con 1 y $2 \mathrm{~mm}$ de diámetro de aspersor en el difusor usando la mezcla de $\mathrm{CO}_{2}$.

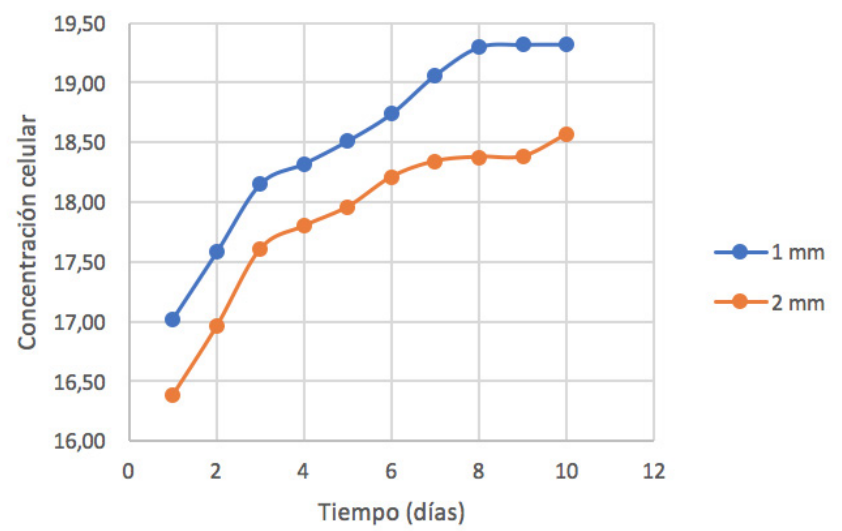

Figura 7. Curvas de crecimiento con $\mathrm{CO}_{2}$ a diferentes diámetros de aspersor Fuente: elaboración propia.

Según el trabajo de los investigadores Doucha et al. (2005), el rango de presión mínimo de $\mathrm{CO}_{2}$ que debe ingresar al cultivo es de 0,1-0,2 $\mathrm{KPa}$, el cual tiene como objetivo evitar que se limite el crecimiento de la microalga por escasez de $\mathrm{CO}_{2}$ y minimizar las emisiones de este compuesto a la atmósfera. Por ello, la presión parcial de $\mathrm{CO}_{2}$ utilizada en esta investigación fue de $0,225 \mathrm{KPa}$, la cual se relaciona con el caudal empleado y explicado en la parte preexperimental.

Los resultados presentados en la tabla 7 permiten apreciar que a menor diámetro de aspersor aumenta la tasa de crecimiento. Esto se debe a que las burbujas con diámetros inferiores se comportan como esferas rígidas con una superficie inmóvil (Doucha \& Lívanskjý, 1998), lo que disminuye la probabilidad de coalescencia entre burbujas y evita que incremente tanto su tamaño como la velocidad con la que asciende (Kadic \& Heindel, 2014), por lo cual se mantienen intactas desde su salida en el difusor hasta la superficie del líquido, contribuyendo a homogeneizar las diferentes zonas dentro del cultivo. Al respecto, cabe 
resaltar que una burbuja que asciende con menor velocidad tendrá un mayor tiempo de residencia en el fotobiorreactor de panel plano (Kadic \& Heindel, 2014).

Por otro lado, Mohsenpour y Willoughby registran tasas de crecimiento menores en comparación con las obtenidas en esta investigación, como se aprecia en la tabla 7. Esta situación se debe al bajo caudal utilizado en nuestro experimento, el cual evitó que la concentración de $\mathrm{CO}_{2}$ empleada $\left(9 \%\right.$ de $\mathrm{CO}_{2}$ ) ocasionara una reducción considerable en el $\mathrm{pH}$, que, como resultado, disminuye la actividad fotosintética e inhibe el crecimiento.

Tabla 7. Tasas de crecimiento con $\mathrm{CO}_{2}$

\begin{tabular}{cccc}
\hline Diámetros $(\mathbf{m m})$ & $\begin{array}{c}\text { Tasa de crecimiento propia }(\mathbf{9} \\
\left.\% \mathrm{CO}_{2}, \text { dias }^{-1}\right)\end{array}$ & $\begin{array}{c}\text { Tasa de crecimiento de } \\
\text { Mohsenpour y Willoughby } \\
{[\mathbf{2 0 1 5}]} \\
\left(5 \% \mathrm{CO}_{2}, \text { dias }^{-1}\right)\end{array}$ & $\begin{array}{c}\text { Tasa de crecimiento de } \\
\text { Mohsenpour y Willoughby } \\
{[\mathbf{2 0 1 5}]\left(\mathbf{1 5} \% \mathrm{CO}_{2}, \text { dias }^{-1}\right)}\end{array}$ \\
\hline 1 & 0,37 & 0,18 & 0,33 \\
\hline 2 & 0,35 & 0 & 0 \\
\hline
\end{tabular}

Fuente: elaboración propia.

Estos valores confirmaron el hecho de que bajo las condiciones del difusor de $1 \mathrm{~mm}$ en el aspersor se obtienen burbujas con mayor área interfacial lo cual conduce a una mayor transferencia de $\mathrm{CO}_{2}$ en las fases gas-líquido (Kadic \& Heindel, 2014).

\section{Planteamiento teórico de las ecuaciones de transferencia de masa en el fotobiorreactor pan- el plano}

Dentro de la revisión bibliográfica realizada se encontró el modelo de la película que permite explicar la transferencia de masa dentro del fotobiorreactor de panel plano, el cual explica la trayectoria del sustrato gaseoso desde la burbuja hasta un organelo en el microorganismo mediante una serie de resistencias.
Al emplear el anterior modelo y utilizar como supuestos teóricos que (i) la fase líquida está a igual temperatura que la fase gaseosa (donde las burbujas tenían un tamaño uniforme y constante); (ii) la presión de $\mathrm{CO}_{2}$ en cada burbuja alcanzaba la presión de saturación; y (iii) que el sistema se encontraba perfectamente mezclado, la transferencia de masa en la interfase gas-líquido como paso limitante a la transferencia de masa general se expresa como se muestra en la ecuación 5 .

La ecuación 5 presenta dos parámetros experimentales (coeficiente de transferencia de masa y área interfacial) que son difíciles de medir por sí solos, por lo que estos se combinan en uno dando lo que se conoce como el coeficiente volumétrico de transferencia de masa [ ( Dutta, 2008).

$$
\frac{P_{C O_{2}}^{\text {burbuja }}}{d t}=-k_{L} a R T\left(\frac{P_{\mathrm{CO}_{2}}^{\text {burbuja }}}{H}-\left[d \mathrm{CO}_{2}\right]\right)
$$

Se debe aclarar que el área interfacial (que hace parte del producto ) se relaciona con el atrapamiento del gas () y el diámetro de la burbuja (), como se aprecia en la ecuación 6. Este parámetro permite explicar los resultados obtenidos en esta investigación utilizando como variable los diámetros de aspersor en el difu- sor (Kadic \& Heindel, 2014), puesto que al disminuir el diámetro de aspersor en el difusor, y por tanto el tamaño de la burbuja, aumenta el área interfacial que se relaciona directamente con el flujo de $\mathrm{CO}_{2}$ desde la fase gaseosa a la fase líquida.

$$
a\left[m^{-1}\right]=\frac{6 \varepsilon}{d_{B}} \quad \text { (Ec. 6) }
$$




\section{CONCLUSIONES}

Los fotobiorreactores de panel plano construidos en láminas de PMMA de 0,008 m presentaron buena resistencia térmica y mecánica. Así mismo, tanto el área de las placas como el volumen del equipo aseguraron una relación superficie/volumen que cumple con rangos establecidos por otros investigadores para garantizar el proceso fotosintético. A su vez, la selección de PVC como material para la elaboración de los difusores garantizó una fácil manipulación y un bajo costo al momento de su implementación en el fotobiorreactor de panel plano.

Los diámetros utilizados en la parte preexperimental corresponden a 1, 2 y $4 \mathrm{~mm}$. Mediante el análisis ANOVA y la prueba $t$ se comprobó que aunque no existe una diferencia significativa en las tasas de crecimiento celular entre estos $\left(0,217\right.$ días $^{-1}, 0,198$ días $^{-1}$ y 0,174 días $^{-1}$, respectivamente) a causa del caudal empleado de 0,17 vvm, la pareja conformada por los diámetros 1 y $2 \mathrm{~mm}$ sí presenta cambios significativos. Por consiguiente, se realizó la parte experimental con estos dos diámetros.

Empleando una mezcla de $9 \% \mathrm{CO}_{2} \mathrm{v} / \mathrm{v}$ y utilizando los diámetros de 1 y $2 \mathrm{~mm}$ durante la fase experimental, se obtuvieron tasas de crecimiento 0,37 días $^{-1}$ y 0,35 días $^{-1}$, respectivamente. Estos resultados demuestran que a menor diámetro de aspersor se obtiene un aumento en la tasa de crecimiento. Así mismo, permiten comprobar que a pesar de utilizar un bajo caudal $(0,17 \mathrm{vvm})$ y una alta presión parcial de $\mathrm{CO}_{2}(0,225 \mathrm{KPa})$, con respecto a investigaciones anteriores, se logra evitar una reducción del $\mathrm{pH}$ y, con ello, disminuir la actividad fotosintética e inhibir el crecimiento.

El modelo de la película junto con los supuestos expresados para el sistema permiten confirmar que el tamaño de la burbuja generado por el aspersor afecta directamente el crecimiento del cultivo. En contraste, se comprueba que una burbuja pequeña con mayor área interfacial, la cual asciende con menor velocidad por el medio de cultivo y por lo tanto tiene un mayor tiempo de residencia en el fotobiorreactor, conduce a una mayor transferencia de masa entre las fases gas-líquido.

\section{REFERENCIAS}

Arredondo, B. O., Voltolina, D., \& Cordero, B. (2007). Concentración, recuento celular y tasa de crecimiento. En B. O. Arredondo, D. Voltolina, T. Zenteno, M. Arce, \& G. A. Gómez (eds.), Métodos y herramientas analíticas en la evaluación de la biomasa microalgal (pp. 25-27). CONACYT.

Doucha, J., \& Lívansjý, K. (1998). Influence of solar irradiance, culture temperature and $\mathrm{CO}_{2}$ supply on daily course of $\mathrm{O}_{2}$ evolution by Chlorella mass cultures in outdoor open thin-layer culture units. Algological Studies, 89, 137-149. https://doi. org/10.1127/algol_stud/89/1998/137

Doucha, J., Straka, F., \& Lívanský, K. (2005). Utilization of flue gas for cultivation of microalgae (Chlorella sp.) in an outdoor open thin-layer photobioreactor. Journal of Applied Phycology, 17, 403-412. https:// doi.org/10.1007/s10811-005-8701-7

Dutta, R. (2008). Fundamentals of Biochemical Engineering. Springer. https://doi. org/10.1007/978-3-540-77901-8

Hernández, G. A. (2014). Evaluación de las incidencias de salinidad y $\mathrm{pH}$ sobre la biomasa, productividad y acumulación de lípidos en cultivos de Chlorella Vulgaris en un fotobiorreactor de placa plana [trabajo de grado, Fundación Universidad de América]. Repositorio Fundación Universidad de América.

Kadic, E., \& Heindel, T. J. (2014). An introduction to bioreactor hydrodynamics and gas-liquid mass transfer. John Wiley \& Sons. https://doi. org/10.1002/9781118869703

Ladislav, N., Cervený, J., Keren, N., \& Kaplan, A. (2010). Experimental validation of a nonequilibrium model of $\mathrm{CO}_{2}$ fluxes between gas, liquid medium, and algae in a flat-panel photobioreactor. Journal of Industrial Microbiology and Biotechnology, 37(12), 1319-1326. https://doi.org/10.1007/ s10295-010-0876-5 
Mohsenpour, S. F., \& Willoughby, N. (2013). Luminescent photobioreactor design for improved algal growth and photosynthetic pigment production through spectral conversion of light. Bioresource Technology, 142, 147-153. https:// doi.org/10.1016/j.biortech.2013.05.024

Mohsenpour, S. F., \& Willoughby, N. (2015). Effect of $\mathrm{CO}_{2}$ aeration on cultivation of microalgae in luminescent photobioreactors. Biomass and Bioenergy, 85, 168-177. https://doi.org/10.1016/j. biombioe.2015.12.002

Rubio-Fernández, D., \& Hernández, G. A. (2016). Evaluación de las incidencias de salinidad y $\mathrm{pH}$ sobre la biomasa, productividad y acumulación de lípidos en cultivos de Chlorella vulgaris en un fotobiorreactor de placa plana. ITECKNE, 13(1), 4456. https://doi.org/10.15332/iteckne.v13i1.1381

Soo, S., \& Choul-Gyun, L. (2003). Photobioreactor engineering: design and performance. Biotechnology and Bioprocess Engineering, 8, 313 321. https://doi.org/10.1007/BF02949274

St-Onge, P. (2013). Optimisation en temps réel de la production de microalgues en photobioréacteur par la commande extrémale. Université du Québec.

Suárez-Chaparro, M. Y., \& Chicagui-Larrota, J. E. (2014). Evaluación de la influencia del dióxido de carbono y la concentración inicial de Chlorella Vulgaris sobre la producción de lípidos a escala laboratorio [trabajo de grado, Fundación Universidad de América]. Repositorio Fundación Universidad de América. 